\title{
Existence of Viscosity Solutions to a Parabolic Inhomogeneous Equation Associated with Infinity Laplacian
}

\author{
Fang Liu \\ Department of Applied Mathematics, School of Science, Nanjing University of Science \& Technology, Nanjing, \\ China \\ Email: sdqdlf78@126.com
}

Received 12 February 2015; accepted 5 May 2015; published 11 May 2015

Copyright (C) 2015 by author and Scientific Research Publishing Inc.

This work is licensed under the Creative Commons Attribution International License (CC BY).

http://creativecommons.org/licenses/by/4.0/

(c) (i) Open Access

\begin{abstract}
In this paper, we obtain the existence result of viscosity solutions to the initial and boundary value problem for a nonlinear degenerate parabolic inhomogeneous equation of the form $u_{t}-\Delta_{\infty} u=f$,
\end{abstract} where $\Delta_{\infty}$ denotes infinity Laplacian given by $\Delta_{\infty} u=\left\langle D^{2} u D u, D u\right\rangle$.

\section{Keywords}

Infinity Laplacian, Viscosity Solution, Inhomogeneous Equation

\section{Introduction}

In this paper, we consider the nonlinear degenerate parabolic inhomogeneous equation involving infinity Laplace

$$
u_{t}-\Delta_{\infty} u=f
$$

where

$$
\Delta_{\infty} u=\left\langle D^{2} u D u, D u\right\rangle
$$

denotes the 3-homogeneous infinity Laplacian. We want to establish the existence result of viscosity solutions to the initial and Dirichlet boundary problem.

The homogeneous infinity Laplace equation $\Delta_{\infty} u=0$ is the Euler-Lagrange equation associated with $L^{\infty}$ variational problem. See for details [1]-[5] and the references therein. Recently, Juutinen and Kawohl [6] con-

How to cite this paper: Liu, F. (2015) Existence of Viscosity Solutions to a Parabolic Inhomogeneous Equation Associated with Infinity Laplacian. Journal of Applied Mathematics and Physics, 3, 488-495.

http://dx.doi.org/10.4236/jamp.2015.35061 
sidered the degenerate and singular parabolic equation

$$
u_{t}=\frac{1}{|D u|^{2}} \Delta_{\infty} u
$$

They proved the existence and uniqueness for both Dirichlet and Cauchy problems, established interior and boundary Lipschitz estimates and a Harnack inequality, and also provided numerous explicit solutions. Due to the degeneracy and the singularity of the Equation (2), they introduced the approximating equations to obtain the existence result with the aid of the uniform continuity estimates. And in [7] we considered the corresponding inhomogeneous parabolic equation. Notice that the 1-homogeneous infinity Laplacian

$$
\frac{1}{|D u|^{2}} \Delta_{\infty} u
$$

is related to game theory named tug-of-war [8]. In [9]-[12], Akagi, Suzuki, et al. considered the following degenerate parabolic equation

$$
u_{t}=\Delta_{\infty} u \text {. }
$$

They also introduced the corresponding approximating equations and got the uniform continuity estimates of approximate solutions by the barrier function arguments. By this approximate procedure, the existence of the solutions was obtained. They also proved the uniqueness and the asymptotic behavior of the viscosity solutions. In [13], Portilheiro and Vázquez considered the parabolic equation

$$
u_{t}=\frac{1}{|D u|^{h}} \Delta_{\infty} u
$$

with $h<2$. They proved existence and uniqueness of viscosity solutions and derived the asymptotic behavior of the solutions for the Cauchy problem and the initial and Dirichlet problem with zero boundary conditions. In [14], Portilheiro and Vázquez studied the nonlinear porous medium type equation involving the infinity Laplacian operator

$$
u_{t}=\Delta_{\infty}^{N}\left(u^{m}\right) .
$$

By the density-to-pressure transformation, they transformed the Equation (4) into a new equation, then the existence, uniqueness and asymptotic behavior etc. were obtained.

In this article, we are interested in the parabolic version of the infinity Laplacian here. We think that Equation (1) is interesting, because it not only is degenerate, but also has many applications in image processing and optimal transportation etc. The parabolic equation involving infinity Laplacian operator has received a lot of attention in the last decade, notably due to its application to image processing, the main usage being in the reconstructions of damaged digital images [15]. For numerical purposes it has been necessary to consider also the evolution equation corresponding to the infinity Laplace operator. We prove the existence of viscosity solutions to the initial-Dirichlet problem by approximating procedure. The approximation process is introduced in [6] for the infinity Laplacian evolution and followed in [9] [13] [14] etc.

This paper is organized in the following order. In Section 2, we give the notations, definitions of viscosity solutions related to the Equation (1). In Section 3, we prove our main existence result by approximating procedure.

\section{Preliminaries}

Throughout of this paper, we use the following notation: If $\Omega \subset R^{n}, Q_{T}=\Omega \times\{0, T\}, \partial_{l} Q_{T}=\partial \Omega \times[0, T)$ denotes the lateral bouncary, $\partial_{b} Q_{T}=\Omega \times\{t=0\}$ the bottom boundary, and $\partial_{p} Q_{T}=\partial_{l} Q_{T} \cup \partial_{b} Q$ (the parabolic boundary of $\left.Q_{T}\right) . \lambda_{\max }(\cdot)$ and $\lambda_{\min }(\cdot)$ denote the largest and the smallest of the eigenvalues to a symmetric matrix. $C^{2,1}\left(Q_{T}\right)$ denotes those functions which are twice differentiable in $x$ and once in $t$.

In the following paper, we adopt the definition of viscosity solutions, (see for example [16]).

Definition 2.1. Suppose that $u: Q_{T} \rightarrow R$ is upper semi-continuous. If for every $\left(x_{0}, t_{0}\right) \in Q_{T}$ and $C^{2,1}\left(Q_{T}\right)$ test function $\varphi$ such that $u-\varphi$ has a strict local maximum at point $\left(x_{0}, t_{0}\right)$, that is $u\left(x_{0}, t_{0}\right)=\varphi\left(x_{0}, t_{0}\right)$ 
and $u(x, t)<\varphi(x, t)$ in a neighborhood of $\left(x_{0}, t_{0}\right)$, there holds

$$
\varphi_{t}\left(x_{0}, t_{0}\right)-\Delta_{\infty} \varphi\left(x_{0}, t_{0}\right) \leq f\left(x_{0}, t_{0}\right),
$$

then we say that $u$ is a viscosity sub-solution of (1).

Similarly, $u: Q_{T} \rightarrow R$ is lower semi-continuous. If for every $\left(x_{0}, t_{0}\right) \in Q_{T}$ and $C^{2,1}\left(Q_{T}\right)$ test function $\varphi$ such that $u-\varphi$ has a strict local minimum at point $\left(x_{0}, t_{0}\right)$, there holds

$$
\varphi_{t}\left(x_{0}, t_{0}\right)-\Delta_{\infty} \varphi\left(x_{0}, t_{0}\right) \geq f\left(x_{0}, t_{0}\right)
$$

then we say that $u$ is a viscosity super-solution of (1).

If $u \in C\left(Q_{T}\right)$ is both a viscosity sub-solution and a viscosity super-solution, then we say that $u$ is a viscosity solution of (1).

\section{Existence Theorem}

In this section we will prove the existence of viscosity solutions to (1) with the initial and boundary data $g$. The method we adopt is the approximation procedure introduced in [6] and used in [9] [13] [14] etc. The main existence result we obtain is.

Theorem 3.1. Let $Q_{T}=\Omega \times(0, T)$, where $\Omega \subset R^{n}$ is a bounded domain, $f$ is continuous in $\bar{Q}_{T}$, and let $g \in C\left(R^{n+1}\right)$. Then there exists a function $u \in C\left(\bar{Q}_{T}\right)$ such that $u=g$ on $\partial_{p} Q_{T}$ and

$$
u_{t}-\Delta_{\infty} u=f
$$

in $Q_{T}$ in the viscosity sense.

We use the approximate procedure, cf, [6] [9] [14]. We consider the approximating equations

$$
u_{t}-\mathcal{L}^{\varepsilon} u=f,
$$

where

$$
\mathcal{L}^{\varepsilon} u=\left(\varepsilon+|D u|^{2}\right) \Delta u+\left\langle D^{2} u D u, D u\right\rangle
$$

with $0<\varepsilon<1$. For this equation with smooth initial and boundary data $g(x, t)$, the existence of a smooth solution $u_{\varepsilon}$ is guaranteed by classical results in [17]. Our goal is to obtain a solution of (1) as a limit of these functions as $\varepsilon \rightarrow 0$. This amounts to proving uniform estimates for $u_{\varepsilon}$ that are independent of $0<\varepsilon<1$. The estimates we require will be obtained by using the standard barrier method.

Theorem 3.2. (Boundary regularity at $t=0)$ Let $Q_{T}=\Omega \times(0, T)$, where $\Omega \subset R^{n}$ is a bounded domain, $f$ is continuous in $\bar{Q}_{T}$, and let $g \in C^{2,1}\left(\bar{Q}_{T}\right)$. Suppose that $u=u_{\varepsilon}$ is a smooth solution satisfying

$$
\begin{cases}u_{t}-\mathcal{L}^{\varepsilon} u=f, & \text { in } Q_{T}, \\ u=g, & \text { on } \partial_{p} Q_{T} .\end{cases}
$$

Then there exists a constant $C \geq 0$ depending on $\left\|g_{t}\right\|_{\infty},\left\|D^{2} g\right\|_{\infty}$ and $\|f\|_{\infty}$ but independent of $0<\varepsilon<1$ such that

$$
|u(x, t)-g(x, 0)| \leq C t \text { in } Q_{T} .
$$

Moreover, if $g$ is only continuous in $x$ (possibly discontinuous in $t$ ) and bounded in $t$, then the modulus of continuity of $u$ on $\Omega \times[0, \bar{T}]$ (for small $\bar{T}$ ) can be estimated in terms of $\|g\|_{\infty},\|f\|_{\infty}$ and the modulus of continuity of $g(x, 0)$ in $x$.

Proof. Step 1. Suppose first that $g \in C^{2,1}\left(\bar{Q}_{T}\right)$ and we consider the upper barrier function

$$
w^{+}(x, t)=g(x, 0)+\lambda t,
$$

where $\lambda>0$ is to be determined. We have

$$
\begin{aligned}
w_{t}^{+}-\mathcal{L}^{\varepsilon} w^{+} & =\lambda-\left[\varepsilon \Delta g(x, 0)+\left\langle D^{2} g(x, 0) D g(x, 0), D g(x, 0)\right\rangle\right] \\
& \geq \lambda-(1+\varepsilon n)\left\|D^{2} g(x, 0)\right\|_{\infty} \geq\|f\|_{\infty} \geq f(x, t),
\end{aligned}
$$


if $\lambda \geq(1+n)\left\|D^{2} g\right\|_{\infty}+\|f\|_{\infty}$. Therefore $w^{+}$is a super-solution.

Clearly, $w^{+}(x, 0)=g(x, 0)$ for all $x \in \Omega$. Moreover, for $x \in \partial \Omega$ and $t>0$,

$$
w^{+}(x, t)=g(x, 0)+\lambda t \geq g(x, t)+\left(\lambda-\left\|g_{t}\right\|_{\infty}\right) t \geq g(x, t),
$$

if $\lambda \geq\left\|g_{t}\right\|_{\infty}$, That is, $w^{+} \geq u$ on $\partial_{p} Q_{T}$.

Thus, by the classical comparison principle, we obtain

$$
u(x, t) \leq w^{+}(x, t)=g(x, 0)+\lambda t
$$

for every $(x, t) \in Q_{T}$. Similarly, by considering also the lower barrier function

$$
w^{-}(x, t)=g(x, 0)-\lambda t,
$$

we obtain the symmetric inequality, and hence the Lipschitz estimate

$$
|u(x, t)-g(x, 0)| \leq C t
$$

for $0<t<T$ and

$$
C=\max \left\{\left\|g_{t}\right\|_{\infty},(1+n)\left\|D^{2} g\right\|_{\infty}+\|f\|_{\infty}\right\} .
$$

Step 2. Suppose now that $g$ is only continuous in $x$ and let $\mu(\cdot)$ be its modulus of continuity. Let us fix a point $x_{0} \in \Omega$ and $0<\tau<\min \left\{\operatorname{dist}\left(x_{0} . \partial \Omega\right), 2 \sqrt{\|g\|_{\infty}}\right\}$. Let us consider the smooth functions

$$
g^{ \pm}(x, t)=g\left(x_{0}, 0\right) \pm \mu(\tau) \pm \frac{2\|g\|_{\infty}}{\tau^{2}}\left|x-x_{0}\right|^{2} .
$$

It is easy to check that $g^{-} \leq g \leq g^{+}$on the parabolic boundary $\partial_{p} Q_{T}$.

Thus if $u^{ \pm}$are the unique classical solutions to (7) with boundary and initial data $g^{ \pm}$, respectively, we have $u^{-} \leq u \leq u^{+}$in $Q_{T}$ by the classical comparison principle again. Since $g^{ \pm}$are smooth, we can use estimate (9) to conclude that

$$
\left|u^{ \pm}\left(x_{0}, t\right)-g^{ \pm}\left(x_{0}, 0\right)\right| \leq \bar{C} t,
$$

where $\bar{C}$ depends on $\tau,\|f\|_{\infty}$ and $\|g\|_{\infty}$. Therefore,

$$
\begin{aligned}
& \left|u\left(x_{0}, t\right)-g\left(x_{0}, 0\right)\right| \\
& \quad \leq\left|u\left(x_{0}, t\right)-u^{ \pm}\left(x_{0}, t\right)\right|+\left|u^{ \pm}\left(x_{0}, t\right)-g^{ \pm}\left(x_{0}, 0\right)\right|+\left|g^{ \pm}\left(x_{0}, 0\right)-g\left(x_{0}, 0\right)\right| \\
& \quad \leq\left|u^{+}\left(x_{0}, t\right)-u^{-}\left(x_{0}, t\right)\right|+\bar{C} t+\mu(\tau) \\
& \quad \leq\left|u^{+}\left(x_{0}, t\right)-g^{+}\left(x_{0}, 0\right)\right|+\left|g^{+}\left(x_{0}, 0\right)-g^{-}\left(x_{0}, 0\right)\right|+\left|g^{-}\left(x_{0}, 0\right)-u^{-}\left(x_{0}, t\right)\right|+\bar{C} t+\mu(\tau) \\
& \quad \leq 3(\bar{C} t+\mu(\tau))
\end{aligned}
$$

with this inequality it is straightforward to complete the proof.

The full Lipschitz estimate in time now follows easily with the aid of the comparison principle and the fact that the Equation (7) is translation invariant.

Corollary 3.3. (Lipschitz regularity in time) If $f$ is continuous in $\bar{Q}_{T}, g \in C^{2,1}\left(\bar{Q}_{T}\right)$ and $u=u_{\varepsilon}$ is as in Theorem 3.2, then there exists a constant $C \geq 0$ depending on $\|f\|_{\infty},\left\|g_{t}\right\|_{\infty}$ and $\left\|D^{2} g\right\|_{\infty}$ but independent of $0<\varepsilon \leq 1$ such that

$$
|u(x, t)-u(x, s)| \leq C|t-s|
$$

for all $x \in \Omega$ and $t, s \in(0, T)$. Moreover, if $g$ is only continuous, then the modulus of continuity of $u$ on $Q_{T}$ can be estimated in terms of $\|g\|_{\infty},\|f\|_{\infty}$ and the modulus of continuity of $g$.

Proof. Let $\tilde{u}(x, t)=u(x, t+\sigma), \quad \sigma>0$. Then both $u$ and $\tilde{u}$ are smooth solutions to (7) in $Q_{\sigma}=\Omega \times(0, T-\sigma)$, 
and hence if $g \in C^{2,1}\left(\bar{Q}_{T}\right)$, we have

$$
\begin{aligned}
\sup _{Q_{\sigma}}|u-\tilde{u}| & =\sup _{\partial_{p} Q_{\sigma}}|u-\tilde{u}| \\
& \leq \max \left\{\|u(\cdot, \sigma)-u(\cdot, 0)\|_{\infty}, \sup _{x \in \partial \Omega}\|u(x, \cdot+\sigma)-u(x, \cdot)\|_{(0, T-\sigma)}\right\} \\
& \leq \max \left\{C \sigma,\left\|g_{t}\right\|_{\infty} \sigma\right\}=C \sigma
\end{aligned}
$$

by the classical comparison principle and Theorem 3.2. This implies the Lipschitz estimate asserted above, and the proof for the case when $g$ is only continuous is analogous.

Theorem 3.4. (Hölder regularity at the lateral boundary) Let $Q_{T}=\Omega \times(0, T)$, where $\Omega \subset R^{n}$ is a bounded domain, $f$ is continuous in $\bar{Q}_{T}$, and let $g \in C^{2,1}\left(\bar{Q}_{T}\right)$. Suppose that $u=u_{\varepsilon}$ is a smooth solution satisfying

$$
\begin{cases}u_{t}-\mathcal{L}^{\varepsilon} u=f, & \text { in } Q_{T}, \\ u=g, & \text { on } \partial_{p} Q_{T} .\end{cases}
$$

Then for each $0<\alpha<1$, there exists a constant $C_{*} \geq 1$ depending on $\alpha,\|g\|_{\infty},\left\|g_{t}\right\|_{\infty},\|f\|_{\infty}$ and $\|D g\|_{\infty}$ but independent of $\varepsilon$ and $\delta$ sufficiently small such that

$$
\left|u\left(x, t_{0}\right)-g\left(x_{0}, t_{0}\right)\right| \leq C_{*}\left|x-x_{0}\right|^{\alpha},
$$

for all $\left(x_{0}, t_{0}\right) \in \partial \Omega \times(0, T)$ and $x \in \Omega \cap B_{1}\left(x_{0}\right)$.

Proof. Step 1. For every $\left(x_{0}, t_{0}\right) \in \partial \Omega \times(0, T)$ and $0<\alpha<1$, let

$$
w^{+}(x, t)=g\left(x_{0}, t_{0}\right)+C_{*}\left|x-x_{0}\right|^{\alpha}+\lambda\left(t_{0}-t\right),
$$

where $C_{*} \geq 1, \lambda>0$ are to be determined. Then a straightforward computation gives

$$
w_{t}^{+}-\mathcal{L}^{\varepsilon} w^{+}=-\lambda-C_{*}^{2} \alpha\left|x-x_{0}\right|^{\alpha-2}\left[C_{*}\left(\varepsilon+\alpha^{2} C_{*}^{2}\left|x-x_{0}\right|^{2 \alpha-2}\right)(n+\alpha-2)+C_{*}^{2} \alpha^{2}(\alpha-1)\left|x-x_{0}\right|^{2 \alpha-2}\right] .
$$

If $\left|x-x_{0}\right| \leq 1$ and $C_{*} \geq 1$, we have

$$
-\varepsilon(n+\alpha-2)-\alpha^{2}(\alpha-1) \geq \alpha^{2} \frac{1-\alpha}{10} .
$$

Therefore

$$
w_{t}^{+}-\mathcal{L}^{\varepsilon} w^{+} \geq-\lambda+C_{*}^{2} \alpha\left|x-x_{0}\right|^{\alpha-2} \cdot \frac{\alpha^{2}(1-\alpha)}{10} \geq-\lambda+C_{*}^{2} \alpha^{3} \frac{1-\alpha}{10} \geq\|f\|_{\infty} \geq f(x, t),
$$

if $C_{*}^{2} \geq \frac{10}{\alpha^{3}(1-\alpha)}\left(1+\lambda+\|f\|_{\infty}\right)$.

We have shown that $w^{+}$is a super-solution of (7).

Step 2. Let $Q_{*}=\left(\Omega \cap B_{1}\left(x_{0}\right)\right) \times\left(t_{0}-t_{*}, t_{0}\right)$, where $t_{*}=\min \left\{1, t_{0}\right\}$. We want to prove first $w^{+} \geq u$ on $\partial_{l} Q^{*}$. Case 1. If $x \in \partial \Omega \cap B_{1}\left(x_{0}\right)$, then

$$
\begin{aligned}
w^{+}(x, t) & =g\left(x_{0}, t_{0}\right)+C_{*}\left|x-x_{0}\right|^{\alpha}+\lambda\left(t_{0}-t\right) \\
& \geq g\left(x_{0}, t_{0}\right)+C_{*}\left|x-x_{0}\right|+\lambda\left(t_{0}-t\right) \\
& \geq-\left\|g_{t}\right\|_{\infty}\left(t_{0}-t\right)-\|D g\|_{\infty}\left|x-x_{0}\right|+g(x, t)+C_{*}\left|x-x_{0}\right|+\lambda\left(t_{0}-t\right) \\
& \geq g(x, t)=u(x, t),
\end{aligned}
$$

provided $C_{*} \geq\|D g\|_{\infty}$ and $\lambda \geq\left\|g_{t}\right\|_{\infty}$.

Case 2. If $x \in \Omega \cap \partial B_{1}\left(x_{0}\right)$, it is easy to see that $v(x, t)=\|f\|_{\infty} t+\|g\|_{\infty}$ is a super-solution of (7) in $Q_{T}$ and $v \geq g$ on $\partial_{p} Q_{T}$. Hence, we have 


$$
\begin{aligned}
w^{+}(x, t) & =g\left(x_{0}, t_{0}\right)+C_{*}+\lambda\left(t_{0}-t\right) \geq g\left(x_{0}, t_{0}\right)+C_{*} \\
& \geq\|g\|_{\infty}+\|f\|_{\infty} T \geq\|g\|_{\infty}+\|f\|_{\infty} t \\
& =v(x, t) \geq u(x, t),
\end{aligned}
$$

provided $C_{*} \geq 2\|g\|_{\infty}+\|f\|_{\infty} T$, and in the last inequality we have used the comparison principle.

Step 3. To prove $w^{+} \geq u$ on $\partial_{b} Q^{*}$.

Case 1. If $t_{*}=t_{0}$, then $Q_{*}=\left(\Omega \cap B_{1}\left(x_{0}\right)\right) \times\left(0, t_{0}\right)$, and notice that since $u=g$ on the bottom of this cylinder,

$$
\begin{aligned}
w^{+}(x, 0) & =g\left(x_{0}, t_{0}\right)+C_{*}\left|x-x_{0}\right|^{\alpha}+\lambda t_{0} \geq g\left(x_{0}, t_{0}\right)+C_{*}\left|x-x_{0}\right|+\lambda t_{0} \\
& \geq g\left(x_{0}, t_{0}\right)+\|D g\|_{\infty}\left|x-x_{0}\right|+\left\|g_{t}\right\| t_{0} \geq g(x, 0),
\end{aligned}
$$

if $C_{*} \geq\|D g\|_{\infty}$ and $\lambda \geq\left\|g_{t}\right\|_{\infty}$.

Case 2. If $t_{*}=t_{0}-1$, then $Q_{*}=\left(\Omega \cap B_{1}\left(x_{0}\right)\right) \times\left(t_{0}-1, t_{0}\right)$. Using the comparison principle again, we have

$$
\begin{aligned}
w^{+}\left(x, t_{0}-1\right) & =g\left(x_{0}, t_{0}\right)+C_{*}\left|x-x_{0}\right|^{\alpha}+\lambda \geq g\left(x_{0}, t_{0}\right)+\lambda \\
& \geq\|g\|_{\infty}+\|f\|_{\infty}(T-1) \geq\|g\|_{\infty}+\|f\|_{\infty}\left(t_{0}-1\right)=v\left(x, t_{0}-1\right) \geq u\left(x, t_{0}-1\right),
\end{aligned}
$$

if $\lambda \geq 2\|g\|_{\infty}+\|f\|_{\infty}(T-1)$.

Step 4. In conclusion, we have shown that $w^{+} \geq u$ on $\partial_{p} Q_{*}$, if we choose

$$
\begin{gathered}
\lambda \geq \max \left\{\left\|g_{t}\right\|_{\infty}, 2\|g\|_{\infty}+\|f\|_{\infty}(T-1)\right\}, \\
C_{*} \geq \max \left\{\|D g\|_{\infty}, 2\|g\|_{\infty}+\|f\|_{\infty} T, \frac{10}{\alpha(1-\alpha)}\left(1+\lambda+\|f\|_{\infty}\right)\right\} .
\end{gathered}
$$

Therefore, we have $w^{+} \geq u$ in $Q_{*}$ by the comparison principle. In particular,

$$
u\left(x, t_{0}\right) \leq w^{+}\left(x, t_{0}\right)=g\left(x_{0}, t_{0}\right)+C_{*}\left|x-x_{0}\right|^{\alpha}
$$

for $x \in \Omega \cap B_{1}\left(x_{0}\right)$. Using the lower barrier

$$
w^{-}(x, t)=g\left(x_{0}, t_{0}\right)-C_{*}\left|x-x_{0}\right|^{\alpha}-\lambda\left(t_{0}-t\right),
$$

we get the symmetric inequality. This finishes the proof.

Due to the translation invariant of the equation and the comparison principle, we can extend the Hölder estimate to the interior of the domain, cf. [6] [14] etc.

Corollary 3.5. (Hölder regularity in space) Let $Q_{T}=\Omega \times(0, T)$, where $\Omega \subset R^{n}$ is a bounded domain, $f$ is continuous in $\bar{Q}_{T}$, and let $g \in C^{2,1}\left(\bar{Q}_{T}\right)$. Suppose that $u=u_{\varepsilon}$ a smooth solution satisfying

$$
\begin{cases}u_{t}=\mathcal{L}^{\varepsilon} u, & \text { in } Q_{T}, \\ u=g, & \text { on } \partial_{p} Q_{T} .\end{cases}
$$

Then there exists $0<\alpha<1$, and constants $C \geq 1$, depending on $\alpha,\|f\|_{\infty},\|g\|_{\infty},\left\|g_{t}\right\|_{\infty}$ and $\|D g\|_{\infty}$ but independent of $\varepsilon$ and $\delta$ sufficiently small such that

$$
|u(x, t)-u(y, t)| \leq C|x-y|^{\alpha},
$$

for all $(x, y) \in \Omega$.

Proof. Step 1. For fixed $0<t<T$, take a point $z \in B_{1}(0)$ and let $V=\Omega \cap \Omega_{z}$. Define $u_{z}(x, t)=u(x-z, t)$. By Theorem 3.4 we have that $\left|u(x, t)-u_{z}(x, t)\right| \leq \bar{C}|z|^{\alpha}$ on $\partial_{l}(V \times(0, T))$ (noting that in this case $x \in \Omega$ or $x-z \in \Omega)$. Hence $\left|u(x, t)-u_{z}(x, t)\right| \leq \bar{C}|z|^{\alpha}$ for every $x \in V$ by the comparison principle. This means that whenever $x, y \in \Omega \cap \Omega_{x-y}$ or $x, y \in \Omega \cap \Omega_{y-x}$ with $|x-y| \leq r$, we have $|u(x, t)-u(y, t)| \leq \bar{C}|x-y|^{\alpha}$.

Step 2. When $|x-y| \geq 1$, using the comparison principle we get 


$$
|u(x, t)-u(y, t)| \leq 2\|g\|_{\infty} \leq 2\|g\|_{\infty}|x-y|^{\alpha}=C|x-y|^{\alpha} .
$$

This finishes the proof.

The following theorem shows that one can obtain the Lipschitz estimate when one remove the Laplacian term from the equation, cf. [6].

Theorem 3.1 follows now easily from Theorem 3.2 and 3.3 and the stability properties of viscosity solutions.

Proof. (Proof of Theorem 3.1) If $g \in C^{2,1}\left(\bar{Q}_{T}\right)$ and $u_{\varepsilon}$ is the unique smooth solution to

$$
\begin{cases}u_{t}-\mathcal{L}^{\varepsilon} u=f(x, t), & \text { in } Q_{T}, \\ u=g, & \text { on } \partial_{p} Q_{T},\end{cases}
$$

Corollaries 3.3 and 3.5 and the comparison principle imply that the family of functions $\left\{u_{\varepsilon}\right\}$ is equicontinuous and uniformly bounded. Therefore, up to a subsequence, $u_{\varepsilon} \rightarrow u$ as $\varepsilon \rightarrow 0$ and $u$ is the unique viscosity solution to (7) by the stability properties of viscosity solutions.

The existence for a general continuous data $g$ follows by approximating the data by smooth functions and using Corollaries 3.3 and 3.5 and the stability properties of viscosity solutions again.

\section{Acknowledgements}

The author would like to thank the anonymous referee for some valuable suggestions.

\section{Support}

This work is supported by the National Natural Science Foundation of China, No.11171153.

\section{References}

[1] Aronsson, G. (1965) Minimization Problems for the Functional $\sup _{x} F\left(x, f(x), f^{\prime}(x)\right)$. Arkiv för Matematik, 6, 3353. http://dx.doi.org/10.1007/BF02591326

[2] Aronsson, G. (1966) Minimization Problems for the Functional $\sup _{x} F\left(x, f(x), f^{\prime}(x)\right)$. II. Arkiv för Matematik, 6, 409-431.

[3] Crandall, M.G., Evans, L.C. and Gariepy, R.F. (2001) Optimal Lipschitz Extensions and the Infinity Laplacian. Calculus of Variations and Partial Differential Equations, 13, 123-139.

[4] Jensen, R. (1993) Uniqueness of Lipschitz Extensions: Minimizing the Sup Norm of the Gradient. Archive for Rational Mechanics and Analysis, 123, 51-74. http://dx.doi.org/10.1007/BF00386368

[5] Aronsson, G., Crandall, M. and Juutinen, P. (2004) A Tour of the Theory of Absolute Minimizing Functions. Bulletin of the AMS, 41, 439-505. http://dx.doi.org/10.1090/S0273-0979-04-01035-3

[6] Juutinen, P. and Kawohl, B. (2006) On the Evolution Governed by the Infinity Laplacian. Mathematische Annalen, 335, 819-851. http://dx.doi.org/10.1007/s00208-006-0766-3

[7] Liu, F. and Yang, X.P. (2015) Viscosity Solutions to a Parabolic Inhomogeneous Equation Associated with Infinity Laplacian. Acta Mathematica Sinica, English Series, 31, 255-271. http://dx.doi.org/10.1007/s10114-015-3244-6

[8] Peres, Y., Schramm, O., Sheffield, S. and Wilson, D. (2009) Tug of War and the Infinity Laplacian. Journal of the American Mathematical Society, 22, 167-210. http://dx.doi.org/10.1090/S0894-0347-08-00606-1

[9] Akagi, G. and Suzuki, K. (2007) On a Certain Degenerate Parabolic Equation Associated with the Infinity-Laplacian. Discrete and Continuous Dynamical Systems, Supplement, 18-27.

[10] Akagi, G. and Suzuki, K. (2008) Existence and uniqueness of viscosity solutions for a degenerate parabolic equation associated with the infinity-Laplacian. Calculus of Variations and Partial Differential Equations, 31, 457-471. http://dx.doi.org/10.1007/s00526-007-0117-6

[11] Akagi, G., Juutinen, P. and Kajikiya, R. (2009) Asymptotic Behavior of Viscosity Solutions for a Degenerate Parabolic Equation Associated with the Infinity-Laplacian. Mathematische Annalen, 343, 921-953. http://dx.doi.org/10.1007/s00208-008-0297-1

[12] Laurencot, P. and Stinner, C. (2010) Refined Asymptotics for the Infinite Heat Equation with Homogeneous Dirichlet Boundary Conditions. Communications in Partial Differential Equations, 36, 532-546.

http://dx.doi.org/10.1080/03605302.2010.498493 
[13] Portilheiro, M. and Vázquez, J.L. (2012) Degenerate Homogeneous Parabolic Equations Associated with the InfinityLaplacian. Calculus of Variations and Partial Differential Equations, 31, 457-471. http://dx.doi.org/10.1007/s00526-012-0500-9

[14] Portilheiro, M. and Vázquez, J.L. (2012) A Porous Medium Equation Involving the Infinity-Laplacian, Viscosity Solutions and Asymptotic Behaviour. Communications in Partial Differential Equations, 37, 753-793. http://dx.doi.org/10.1080/03605302.2012.662665

[15] Caselles, V., Morel, J.M. and Sbert, C. (1998) An Axiomatic Approach to Image Interpolation. IEEE Transactions on Image Processing, 7, 376-386. http://dx.doi.org/10.1109/83.661188

[16] Crandall, M.G., Ishii, H. and Lions, P.L. (1992) User's Guide to Viscosity Solutions of Second-Order Partial Differential Equations. Bulletin of the AMS, 27, 1-67. http://dx.doi.org/10.1090/S0273-0979-1992-00266-5

[17] Ladyženskaya, O.A., Solonnikov, V.A. and Ural'ceva, N.N. (1967) Linear and Quasilinear Equations of Parabolic Type, Translations of Mathematical Monographs, Vol. 23, American Mathematical Society, Providence, R.I. 